expliquer les différences profondes constatées entre les formules leucocytaires des laits issus d'une même mamelle; les microbes, selon leur nature et leur virulence, déterminent un appel leucocytaire d'intensité et de qualité variables dans le quartier infeeté. Il n'y a plus uniquement filtration de leucocytes comme dans les quartiers sains, mais une véritable mobilisation des différentes classes actives des défenseurs de l'organisme; la connaissance des mobilisés permettra d'avoir la certitude de l'attaque, c'est-à-dire l'existence d'une infection. L'examen qualitatif des leucocytes, quartier par quartier, peut donc parfaitement servir de base à un contrôle sanitaire du lait.

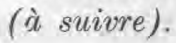

\title{
QUELQUES CONSIDÉRATIONS DE PHYSIOLOGIE SUR LE MÉTABOLISIME DE LA GRAISSE DANS L'ORGANISIME. A PROPOS DE LA LIPO-DIÉRÈSE PULMONAIRE, OU UN ESSAI DE PHYSIO-ZOOTECHNIE.
}

\author{
par J. BLIER, \\ Docteur-Vétérinaire.
}

Dans un livre récent (1), qui a sensiblement le même titre que cette étude, j'ai été conduit à développer quelques considérations relatives au métabolisme des corps gras chez les mammifères grâce au rapprochement des deux faits suivants:

10 La découverte par RogER et BINET de la lipo-diérèse pulmonaire ;

$2^{\circ}$ L'étonnante richesse en crème du lait des cétacés (elle atteint parfois $50 \%$ ).

La découverte de la lipo-diérèse pulmonaire contribue à éclairer le métabolisme des corps gras chez les mammifères non seulement dans le tissu du poumon, mais même dans leur organisme total.

Si ces deux chercheurs ont mis en évidence une diastase capable d'assurer dans tous les parenchymes, et surtout dans l'appareil respiratoire, la destruction des graisses, il est logique, à la lumière de leur travail, de tenter l'interprétation d'autres phénomènes jusqu'ici obscure.

Dans le cas des mammifères marins, on peut se demander si cette diastase destructrice de leurs graisses ne va pas être pertubée dans son action par des agents d'origine marine contenus dans l'air inspiré et dont certains peuvent exercer une action anti-catalysante. Ainsi s'expliquaient peut-être, à mon avis, la tendance bien connue des cétacés à l'obésité, et la non moins curieuse richesse de leur lait en graisse.

Jusqu'ici on n'a jamais pu encore pénétrer le secret qui préside à l'inégalité de la richesse des laits en matière grasse. On est d'accord

(1) Ce livre a exactement pour titre: Un Nouveau facteur PLAUSible d'okésitê. A. propos de la lipo-diérèse pulmonaire ou un Essai de Physio-zootechnie par Jules ButER. L'ouvrage figure dans la Collection des Travaux de Pathologie comparée édités par la Revue de Pathologie comparée et d'Hygiène générale. En vente: 8, rue des Acacias, Paris (17e). Prix: 15 franes. 
toutefois pour reconnaître qu'elle est fonction de la race et de la famille, ce qui permet des sélections avantageuses.

Si nous établissons le tableau des richesses butyreuses moyennes des laits des différentes rases, il semble que nous voyons jouer également l'influence de la mer. En tête, nous notons en effet la race de Jersey, suivie tout de suite après de la Parthenaise, appelée quelquefois Vendéenne, suivie à son tour de la Cotentine.

A l'attrait physiologique de la question s'ajoute un immense intérêt économique.

Le contrôle laitier, qui n'est qu'une statistique beurrière, prouve, par l'intérêt avec lequel l'accueillent les éleveurs avisés, combien la richesse des laits en corps gras fascine, l'agriculture de tous les pays. Da lastose, on en a que trop; de la caséine, on ne sait parfois qu'en faire, alors que du beurre, on en manque toujours.

La notion de physio-zootechnie. - Physiologie et zootechnie, voilà deux mots qui paraissent limpides pour le biologiste. D'ailleurs les zootechniciens semblent tous enclins à réunir leur art à la physiologie. Claude BernaRD avait vu dans la zootechnie, non seulement de la biologie expérimentale, mais aussi, suivant son expression, "comme une sorte de physiologie également expérimentale ".

Le zootechnicien ne peut plus se contenter d'une physiologie de plein air; il lui faut expérimenter.

L'opinion est unanime aujourd'hui, à proclamer l'insuffisance de la zootechnie basée sur la seule étude des formes, et le succès du contrôle laitier le prouve surabondamment.

Faire du contrôle laitier, e'est faire en même temps de la génétique, e'est extraire de l'ensemble des animaux de choix dont on assurera par des croisements appropriés la transmission de qualités exceptionnelles.

Resonnaître la vache Cotentine à ses bringeures, et s'arrêter là, n'est qu'un geste indigent et sans signification. La zootechnie doit être fille de la physiologie, et pour asseoir cette filiation, il suffirait de prouver qu'il y a une physiologie au moins par race, sinon par famille ou même par individu. Or, dans l'ordre des infiniment petits, ceci ne fait pas de doute; la chose paraît moins claire chez les espèces plus élevées en organisation, à fortiori chez les mammifères, mais à priori, il est juste de supposer qu'elle n'existe pas moins. Au surplus, l'idée de sang butyreux émise par le professeur Ch. Porcher et qui nous parait particulièrement féconde et digne de faire l'objet de recherches expérimentales de grande envergure, répond à cette physiologie de rase ou même individuelle à laquelle je faisais allusion tout à l'heure.

Il est un grand nombre de faits, sur lesquels je ne puis insister ici, mais que l'on trouvera dans mon livre, par lesquels se trouve suffisamment affirmée la liaison des caractères ethniques avec la physiologie 
pure, pour engager les zootechniciens à s'évader des frontières que leur trace la morphologie.

N'est-ce pas un de ees faits que celui mis en relief par l'ingénieur agronome B. Macalik? En étudiant les brebis laitières de la Tchécoslovaquie, il tente d'établir " une relation entre les qualités de la laine et les facultés butyreuses. Plus grasse en moyenne est la laine, plus gras est le lait obtenu, toutes conditions égales d'ailleurs »)(1).

II serait par conséquent intéressant de voir, et c'est là une expérience que je propose, si la vache bonne beurrière en moyenne produit, en général, un cérumen plus richè en corps gras, ce qui permettrait, en dosant les corps gras dans le cérumen des taureaux, de les sélectionner afin d'en obtenir des filles bonnes beurrières...

L'histoire des corps gras du lait est tout à fait eaptivante. Ce qu'il faut surtout en retenir, c'est que, parmi la foule des facteurs inconnus dont la graisse du lait semble le jouet, il n'y en a qu'un dont l'éleveur ait pu, jusqu'à présent, retenir l'incontestable influence : c'est l'hérédité.

Pour l'instant, notons l'irrégularité ethnique de la proportion des corps gras dans le lait. Elle est un argument de plus en faveur de l'intérêt de la physio-zootechnie qui réclame de larges expériences, puissamment outillées, faites à la fois dans des stations édifiées à ce sujet et sous le contrôle d'éleveurs intelligents, consciencieux et soigneux, attentifs à suivre le rendement de chacun des animaux de leur cheptel.

La perturbation plausible de la lipo-diérèse pulmonaire chez les animaux laitiers sous l'influence d'anti-catalyseurs contenus dans l'air marin. - C'est de très bonne heure que l'on a remarqué ce qu'on peut appeler l'anarehie butyreuse du lait. Aucune loi jusqu'ici, le facteur hérédité étant laissé de côté, n'a été formulée pour expliquer comment le lait semble dépendre de purs caprices pour sa richesse en corps gras. Nous allons nous efforcer de soulever le voile.

La découverte de Roger et L. Binet. - C'est le travail de ces savants qui domine mon sujet. Ils ont montré en effet, comme le dit lui-même M. le professeur RoGER, "le rôle primordial du poumon dans le métabolisme des matières grasses ".

En effet, tandis que les hydrates de carbone et les albumines provenant de l'alimentation, passent dans les rameaux de la veine porte, pour être amenés dans le foie qui les emmagasine et les transforme, les graisses suivent un tout autre chemin. "Dédoublées par le suc pancréatique, elles se reconstituent dans les parois intestinales, pénètrent dans les chylifères, traversent les ganglions mésentériques et arrivent par le canal thoracique dans la veine sous-clavière gauche, où elles se mélangent au sang veineux pour être lancées par le ventricule droit

(1) Le Lait, $\mathrm{n}^{\circ} 33$, mars 1924, p. 200. 
dans la petite circulation. Le poumon est donc le premier organe qu'elles traversent. Pour Roger et BINET, il s'y trouve un ferment qu'ils appellent lipo-diérase, existant d'ailleurs dans tous les tissus, et qui détruit en partie la matière grasse. L'action de cette diastase dans le parenchyme pulmonaire peut, à son tour, être le jouet d'une action perturbatrice liée à la présence d'anti-ferments, ou, comme on dit en chimie générale, d'anti-eatalyseurs. Comment seraient apportés ceux-ci ? Par l'air introduit dans le poumon et chargé parfois de produits d'origine marine pour les mammifères d'habitat maritime, ou encore chargé de gaz ammoniac pour les animaux soumis par les éleveurs à des conditions très précises d'engraissement.

Un coup d'oeil sur la respiration des cétacés, sur leur appareil laitier et sur la composition chimique de l'eau de mer. - Un cétacé, quand il respire, introduit dans ses poumons un mélange d'air et d'eau de mer, c'est-à-dire un mélange d'air et d'agents anti-catalyseurs capables de perturber la lipo-diérèse.

Or, les cétacés sont de beaucoup les mammifères les plus gras; ce sont eux également qui ont le lait le plus gras.

L'action anti-catalysante exercée par l'eau de mer peut se rattacher aux matières salines présentes dans ce liquide à un taux assez élevé, au chlorure de sodium peut-être, mais aussi aux composés iodés; je n'insisterai d'ailleurs pas sur ce point. En faisant allusion à la richesse du lait des cétacés en matière grasse, il est peut-être bon de formuler une remarque, peut-être grosse de conséquences, c'est que les cétacés qui ont fourni les laits qui ont pu être analysés (1) ont été naturellement traits après leur mort, ce qui soulève la question de la traite postmortem.

Les baleinidés ne sont pas exclusivement cantonnés dans les régions froides. La baleine, à certaines époques régulières, recherche les mers chaudes. C'est au large du Gabon notamment, qu'ont lieu les accouplements et les accouchements.

Si l'eau de mer contient, en dehors du chlorure de sodium, des sels de magnésie, des composés iodés, un grand nombre de corps à l'état infinitésimal, si les cétacés ont un parenchyme pulmonaire au eontact d'un air mêlé d'embruns, le problème est un peu différent pour les femelles bovines, incontestablement exposées aux effets d'un air chargé de produits marins, quand elles habitent un climat placé sous l'influence de la mer, mais échappant néanmoins à l'action directe de l'eau des vagues sur leurs cellules pulmonaires.

Je me bornerai à rappeler que si l'air marin, prélevé sur le littoral, ne contient pas, au sens étroit du mot, des gouttelettes d'eau de mer, il possède en suspension nombre de produits issus de la mer. L'action

(1) Voír le livre de Grrmmer "Chemie und physiologie der Mileh ". Analysé dans Le Lait, $n^{\circ} 61$, p. $111,1926$. 
anti-catalysante, que nous attribuons à l'eau de mer, peut se retrouver sans doute dans l'ammoniac et il est curieux peut-être de rappeler que les éleveurs d'oies savent, dans la région de Toulouse, que pour engraisser à fond une oie, il importe de faire appel à une foule de précautions classiques telles que la suralimentation, la sédentarité, etc..., mais qu'il convient aussi de ne jamais nettoyer la litière; on assure ainsi à l'oie une atmosphère ammoniacale dont on a reconnu l'avantage. On sait également que les moutons s'engraissent davantage dans les locaux dont le sol est parfois recouvert d'une grande épaisseur d'exeréments, véritable source-d'ammoniac.

Rapprochement entre la femelle bovine du type insulaire, du type maritime et les cétacés. - Nous croyons qu'il est possible, chez les laitières de climat maritime, d'invoquer une perturbation de la lipo-diérèse pulmonaire analogue à celle qui paraît probable et plus prononcée chez les baleinoptides.

Nous n'ignorons pas que la thèse que nous soutenons est susceptible de nombreuses objections et la première qui se présente à l'esprit est tirée de la vache hollandaise, animal, qui comme la Jerseyaise, vit près de la mer, mais dont le lait est relativement pauvre en matière grasse. Il est vrai qu'il importe, dans une question comme celle que nous soulevons, non seulement de connaître le taux butyreux moyen d'une race, mais également la production totale de sa graisse au cours d'une lactation.

Or, si la Hollandaise est une faible beurrière, comme on a l'habitude de le dire, e'est parce que l'on ne considère que le rendement par litre; si, au contraire, on envisage le rendement total en lait et qu'on multiplie le nombre de litres secrétés au cours d'une lactation par le taux butyreux moyen, on eonstate que la Hollandaise est de toutes les races bovines celle qui donne la plus grande quantité en valeur absolue de matière grasse au cours d'une lactation.

Une autre objection que l'on peut faire à mon hypothèse, c'est celle qui est relative à la comparaison de la nourrice maigre et de la nourrice grasse. Je n'ai vu mentionner nulle part, malgré des recherches dans lesquelles $m$ 'ont très aimablement aidés des spécialistes qualifiés, une différence entre le lait des femmes grasses et celui des femmes maigres, L'obésité ne semble pas changer la composition du lait de femme; la femme obèse n'a pas un lait plus gras que celui de la femme maigre. La variation en graisse du lait de femme est done, comme celle du lait de vache, anarchique.

Une autre objection peut être tirée du climat maritime envisagé en général. S'il semble établi qu'il y ait un parallélisme entre la richesse butyreuse du lait des cétacés et celle du lait des vaches laitières de climat maritime, peut être ne faut-il point incriminer uniquement la pertur- 
bation de la lipo-diérèse pulmonaire? et peut-être faut-il chercher un autre phénomène marin capable de provoquer l'enrichissement du lait en eorps gras. Il y a en effet un point très obscur dans le peu que nous pouvons connaître de la physiologie des cétacés. C'est l'énigme de leur ravitaillement en eau douce. La rencontrent-ils :

$1^{\circ}$ A l'embouchure des fleuves;

$2^{\circ}$ En provenance de la fusion des glaces;

$3^{\circ}$ Dans les petits mollusques qu'ils avalent en quantité énorme?

Autant de mystères ; ce sont des mammifères, il n'est jamais inutile de le rappeler. Que boivent-ils? on n'en sait rien. Or, il semble que lorsqu'une femelle est privée d'eau, son lait s'enrichit en graisse. En tous cas. l'objection que nous soulevons ici n'est peut-être pas d'une très grande solidité; elle soulève des observations intéressantes, mais elle est incapable d'expliquer la richesse en matière grasse du lait de la vache jerseyaise qui boit à sa soif.

Une autre objection, car il nous plaît de les accumuler, est tirée de la permanence de la valeur butyrogène d'un animal laitier malgré sa transplantation. On sait en effet que si on transporte la vache jerseyaise sur le continent, elle continue, comme ses filles d'ailleurs, à fournir un lait très gras. Les éleveurs Nord-Américains ont créé une variété de Jerseyaises d'un grand format, depuis plusieurs générations déjà, qui leur donne toute satisfaction au point de vue butyreux. L'objection que nous rappelons ici est la moins grave de toutes, car les biologistes connaissent depuis longtemps l'hérédité des caractères acquis.

Je pourrais encore formuler une objection tirée de l'influence probable de la vitamine de croissance (facteurs lipo-solubles A) sur la richesse des laits en matière grasse, mais notre ignorance est encore trop grande du rôle de ces vitamines pour que j'insiste.

Je reconnais que dans ce travail les pages qui précèdent sont au fond une œuvre de pure réflexion. Sans doute, nous n'avons pas abouti à des preuves, mais simplement à des hypothèses de travail que nous croyons plauisbles et qui appellent bien entendu des expériences sur le plan desquelles je n'ai pas à insister, car leursidées directrices découlent des faits que j'ai rassemblés et des réflexions qui les accompagnent.

D'ailleurs j'ai exposé, dans mon ouvrage, diverses expériences à. réaliser, mais sans doute sera-t-il facile à nombre de lecteurs d'en imaginer d'autres.

\section{BIBLIOGRAPHIE}

Je ne puis reproduire ici dans le détail la bibliographie qui accompagne mon travail mais je me fais un devoir de fournir néanmoins quelques indications essentielles.

Sur les curieuses particularités anatomiques de l'appareil mammaire chez les cétacés, voir : BeAuregard et Boulard. Recherches sur les appareils génito-uri- 
naires des Balonidés (1). Journal d'Anatomie et de Physiologie, page 158, année 1882.

Pour le travail fondamental de Roger et Binet sur la lipo-diérèse pulmonaire, voir (édité par Masson) dans leur volume intitulé : Questions actuelles de Biologíe Médicale, leur mémoire qui a pour titre: Les fonctions internes du poumon. Action du poumon sur les matières grasses.

Voir: W, Kopaczewski (ehez l'éditeur Vigot, Paris) Catalyse et applications.

Voir: GrJMmer, Chemie und Physiologie der Milch. Verlagbuchlandlung Paul Parey, Berlin, 1910, page 60.

Voir : MACALIK, Rapport entre la quantité de la matière grasse du lait des brebis, la teneur en graisse et la finesse de leur laine et les facultés laitières, dans la revue "Le Lait ", page 195, n 33, mars 1924.

Voir : A. Lavornne. Les Sociétés de Contrôle laitier en Seine-Inférieuré. Dans la revue "Le Lait $», n^{\circ} 7$ et 8 en 1922.

\section{BIBLIOGRAPHIE ANALYTIQUE}

\section{$1^{\circ}$ LES LIVRES}

\section{Cantor (E.). - Le lait des animaux tuberculeux et ses dangers. -} Etudes expérimentales sur la répartition du bacille de Koch dans les sous-produits de la laiterie. Th. Doct. Vétér,, Paris, 1925.

C'est surtout dans les sous-produits de laiterie, particulièrement riches en globules graisseux, que s'accumulent les germes virulents de la tuberculose. Au fur et à mesure que la fabrication des dérivés lactés tend à produire des substances moins riches en globules butyreux, le danger consécutif à l'ingestion diminue du fait de la raréfaction du bacille tuberculeux. La répartition bacillaire d'après les recherches de l'auteur est la suivante: le fromage gras contient le plus grand nombre de bacilles $(80 \%)$; la crème en contient $65 \%$ et le lait écrémé $33 \%$; le fromage maigre $26 \%$, le petit-lait provenant du fromage gras $15 \%$; enfin le petitlait du fromage maigre contient le plus petit nombre de bacilles, soit $10 \%$. Donc possibilité grande d'infection par le fromage gras, le beurre, la crème; possibilité moindre par le fromage maigre et le lait écrémé,

\section{Gegenheimer (Vida). - Etude sur la détermination quantitative \\ de la cystine dans la caséine. Thèse Doct. Phil. de Columbia University. New-York. 1925.}

Les chiffres qui ont été donnés sur le taux de la cystine dans la caséine sont très variables, ce qui tient à la méthode utilisée. Ms vont de $0,06 \%$ à $1,77 \%$. On dose le soufre de la eystine à l'état de sulfate de baryum.

Différents auteurs : van SLYKÉ, GoRTNER, OsBorNe et autres ont insisté particulièrement sur l'importance du temps employé pour l'hydrolyse. C'est un des facteurs à considérer dans le dosage de la cystine. D'après G., le maximum de la eystine est obtenu au bout de 5 à 6 heures de chauffage à $115^{\circ} \mathrm{C}$. aveo de l'acide

(1) Signalons notamment que les baleines ont les mamelles dans les plis de la vulve Ces organes possèdent des muscles favorisant l'expulsion brusquée du lait et un dispositif protecteur contre l'eau de mer. 